\title{
Dual-incision approach for excision of postauricular sinus
}

\author{
Hiroshi Sakaida $^{1}$ (D) Tomotaka Miyamura $^{1} \cdot$ Yasunori Matsuda $^{1} \cdot$ Kazuhiko Takeuchi $^{1}$
}

Received: 17 August 2015 / Accepted: 28 September 2015 / Published online: 5 October 2015

(C) Springer-Verlag Berlin Heidelberg 2015

Sir,

Preauricular sinus is a common congenital anomaly, which results from developmental failure of embryogenesis during which six hillocks normally fuse to form the auricle. The opening is typically located near the anterior limb of the ascending helix $[1,2]$. However, in rare instances, the opening appears at the crus of the helix, penetrates through the auricular cartilage, and reaches the postauricular area (Fig. 1). This latter type of preauricular sinus is called postauricular sinus [2-4]. Surgery for typical preauricular sinus is usually not complicated because there is minimal involvement of the auricular cartilage. However, special surgical consideration is necessary for postauricular sinus because of the opening penetrating throughout the auricular cartilage. We report here on two patients with postauricular sinus successfully treated by a dual-incision technique.

The surgical procedure was performed as described below, but some modifications were made depending on the case. All surgeries were performed under general anesthesia, and a surgical microscope was used for both the precise identification and the delicate dissection of the sinus tract. A fine lacrimal probe was used to identify the direction of the sinus tract. At the beginning of the surgery, a small amount of crystal violet solution was injected into the lumen of the sinus tract through the opening so that the entire sinus could be clearly identified during the surgery.

Hiroshi Sakaida

hsakaida@clin.medic.mie-u.ac.jp

1 Department of Otorhinolaryngology-Head and Neck Surgery, Mie University Graduate School of Medicine, 2-174 Edobashi, Tsu, Mie 514-8507, Japan
A wedge-shape skin incision was made around the sinus opening at the crus of the helix. The skin inside the incision line was elevated. The cartilage surrounding the sinus tract was minimally excised together with the sinus tract to ensure complete removal of the epithelial lining (Fig. 2). The dissection proceeded as deep as possible until the surgical field was limited. Care was taken to minimize the skin incision and the resection of the auricular cartilage to prevent any postoperative deformity.

A retroauricular incision was then made along the attachment of the auricle. After identifying the distal end of the sinus tract with visual guidance by the crystal violet solution injected at the beginning of the surgery, the dissection was carried out anteriorly along the sinus tract, which was found to penetrate the cartilage (Fig. 3). The sinus tract was finally resected in a bidirectional fashion. After the complete resection of the sinus tract, the dead space was sealed with a layered closure.

One patient was a 17-year-old boy with a history of more than 10 episodes of repeated postauricular swelling (patient 1). A small opening measuring $1 \mathrm{~mm}$ in diameter was visible at the crus of the helix of the left auricle. An induration was noted at the posteriosuperior region of the left auricle, indicating a chronically inflamed distal end of the sinus tract (Fig. 1). The other patient was a 2year-old girl with a history of recurrent swelling at the concha of the left auricle (patient 2). Physical examination revealed a small opening measuring $1 \mathrm{~mm}$ in diameter at the crus of the helix of the auricle. There was no opening at the postauricular area. These patients had uneventful surgical repair of these lesions. At their followups 1 year later, there were no signs of recurrence. There was minimal postoperative deformity of the crus of the helix that was acceptable to both patients.

A dual-incision approach is a useful and reasonable procedure for postauricular sinus. There are three points that require caution when performing this procedure. 


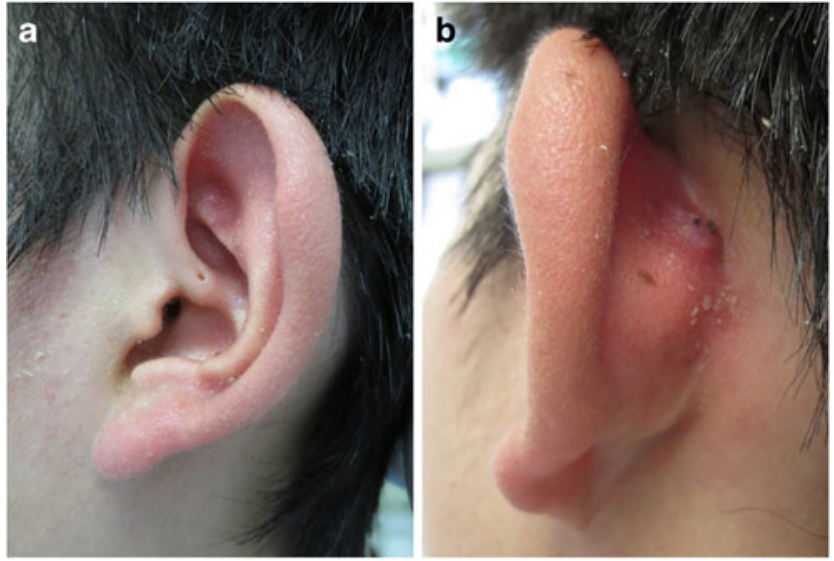

Fig. 1 Patient 1. The opening of the sinus tract located at the crus of the helix of the auricle (a). An induration at the posterior aspect of the auricle (b)

First, the sinus tract is so narrow that it seems to be practically impossible to detach the epithelial lining from the inner surface of the cartilage surrounding the sinus tract. Even if this can be done, there may be a risk of epithelial remnants. Therefore, the cartilage surrounding the sinus tract should be excised together to ensure complete removal of the epithelial lining. Resection of the cartilage of the crus of the helix can be a cause of concern for postoperative deformity, and the amount of the resected cartilage should be minimized.

Second, since the sinus tract dissection proceeds deeply and posteriorly, visualization and identification of the sinus tract becomes difficult from the anterior due to limited view through the small cartilage opening. A wider incision could allow resection of the sinus tract and surrounding cartilage from the anterior view, but this may leave a larger surgical scar. Moreover, the sinus tract may vary in length and in direction [2]. In this situation, an additional retroauricular incision is useful to identify the distal end of the sinus tract. Unlike the incision at the anterior aspect of the auricle, a retroauricular incision is not visible and will not cause a cosmetic problem

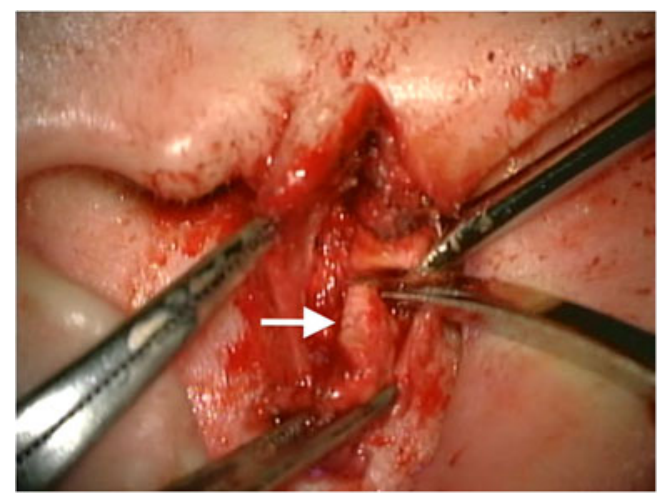

Fig. 2 Patient 1. Intraoperative anterior view showing the anterior half of the sinus tract being dissected together with the surrounding cartilage (arrow)

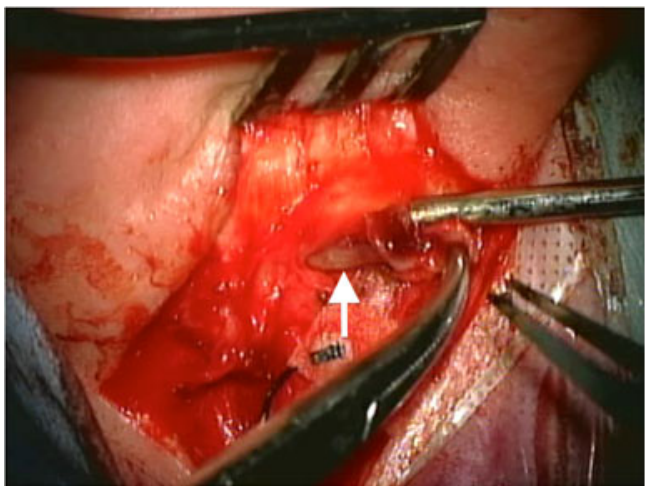

Fig. 3 Patient 1. Intraoperative posterior view showing the posterior half of the sinus tract being dissected anteriorly (arrow). Note that the sinus tract penetrates the auricular cartilage

even if it is wider. Care should be taken not to injure the sinus tract when making a retroauricular incision.

Third, several additional techniques have been advocated for complete resection of the sinus tract. A surgical microscope should be used to achieve a precise dissection [5, 6]. The use of a dye solution has been suggested to identify the sinus tract [7], and a probe is generally used for cannulation of the sinus tract.

Our experience with these cases highlights the dualincision approach coupled with several additional techniques as a useful and reasonable procedure to achieve complete excision of the postauricular sinus.

\section{Compliance with ethical standards}

Conflict of interest Hiroshi Sakaida, Tomotaka Miyamura, Yasunori Matsuda, Kazuhiko Takeuchi declare that they have no conflict of interest.

Ethical standards For this type of study, formal consent from an ethics committee is not required.

Informed consent Informed consent was obtained from all individual participants included in the study.

\section{References}

1. Yeo SW, Jun BC, Park SN, Lee JH, Song CE, Chang KH, Lee DH (2006) The preauricular sinus: factors contributing to recurrence after surgery. Am J Otolaryngol 27(6):396-400

2. Choi SJ, Choung YH, Park K, Bae J, Park HY (2007) The variant type of preauricular sinus: postauricular sinus. Laryngoscope 117(10):1798-1802

3. Ng W, Chew Y, Tan K, Chong A (2011) Pre-auricular sinus: an uncommon presentation. Malays Fam Physician 6(1):32-33

4. Kim HJ, Lee JH, Cho HS, Moon IS (2012) A case of bilateral postauricular sinuses. Korean J Audiol 16(2):99-101

5. Chang PH, Wu CM (2005) An insidious preauricular sinus presenting as an infected postauricular cyst. Int J Clin Pract 59(3):370-372

6. Raman R (1990) Excision of preauricular sinus. Arch Otolaryngol Head Neck Surg 116(12):1452

7. Currie AR, King WW, Vlantis AC, Li AK (1996) Pitfalls in the management of preauricular sinuses. Br J Surg 83(12):1722-1724 